\title{
Chlamydial cervicitis and urethritis: single dose treatment compared with doxycycline for seven days in community based practises
}

Department of Obstetrics and Gynecology, University of

Tennessee, Memphis, Tennessee

E M Thorpe Jr

Department of Medicine, University

of Washington, Seattle, Washington

W E Stamm

Department of

Medicine, University

of Alabama-

Birmingham,

Birmingham, Alabama

E W Hook III

Department of

Obstetrics and

Gynecology,

University of

Louisville, Louisville,

Kentucky

S A Gall

Department of

Medicine, Indiana

University,

Indianapolis, Indiana

$\mathrm{R} B$ Jones

St Paul Division of

Public Heath, St Paul,

Minnesota

K Henry

Department of

Biostatistics,

Pharmaco LSR,

Incorporated, Austin,

Texas

G Whitworth

Pfizer Central

Research, Groton,

Connecticut

R B Johnson

Address correspondence to:

E M Thorpe Jr,

Department of Obstetrics

and Gynecology, University

of Tennessee-Memphis,

1211 Union Avenue, Suite

38104, USA.

Accepted for publication

28 December 1995

\author{
E M Thorpe Jr, W E Stamm, E W Hook III, S A Gall, R B Jones, K Henry, G Whitworth,
} R B Johnson

Study goal: To compare the efficacy and safety of single $1 \mathrm{~g}$ oral azithromycin with doxycycline, $100 \mathrm{mg}$ twice daily for seven days for treatment of uncomplicated urogenital chlamydial infection.

Study design: Randomised, unblinded, comparative trial, involving 597 patients demonstrating clinical evidence of genital chlamydia and a positive non-culture assay for Chlamydia trachomatis. Results: Among the azithromycin- and doxycycline-treated patients $61 \%$ and $60 \%$, respectively, were asymptomatic within one week after the first dose. At two weeks, these figures increased to $86 \%$ and $83 \%$, respectively. Bacteriological eradication, based on a negative assay, occurred in 338 (97\%) of 347 azithromycin-treated patients and 161 (99\%) of 163 doxycyclinetreated patients.

Conclusion: Treatment of uncomplicated chlamydial cervicitis and urethritis with single $1 \mathrm{~g}$ oral azithromycin is equivalent to standard therapy with doxycycline. Drug-related adverse events were approximately twice as common as previously reported for both drugs.

(Genitourin Med 1996;72:93-97)

Keywords: azithromycin; Chlamydia trachomatis; doxycycline

\section{Introduction}

Chlamydia trachomatis is among the most prevalent sexually transmitted diseases in the United States, accounting for an estimated four million cases each year. ${ }^{1}$ These infections are often asymptomatic, but are associated with substantial morbidity in men, women, and infants. This is particularly true for women, in whom the sequelae of chlamydial upper genital tract infection (endometritis and salpingitis) include tubal infertility and ectopic pregnancy. ${ }^{23}$

One impediment to effective treatment of chlamydial infections has been the need for patients to take seven or more days of multidose therapy, usually with antibiotics which have at least moderate gastrointestinal side effects. Consequently, some symptomatic patients are noncompliant, especially after they improve with partial therapy. Compliance with multiple day therapy is even more difficult in asymptomatic patients and may result in treatment failure. Treatment failures may then be responsible for persistent infections and reinfection of sexual partners.

Azithromycin is an azalide antibiotic that has a minimum inhibitory concentration (MIC) against $C$ trachomatis of 0.03 to 0.25 $\mathrm{mg} / 1^{45}$ and achieves high intracellular concentrations, which may be beneficial in eradicating chlamydia, a known obligate intracellular pathogen. ${ }^{6}$ In addition, azithromycin has good in vitro activity against other sexually transmitted pathogens that are often present concurrently, including Ureaplasma urealyticum. ${ }^{7}$ Finally, azithromycin has tissue bioavailability and a tissue half-life of between two and four days. ${ }^{8}$ These pharmacokinetic properties imply that the dosing period for azithromycin can be significantly reduced, while still achieving high antimicrobial activity at sites of infection. Several studies, conducted mainly in academic medical centres using patients attending STD clinics, have demonstrated that an oral single dose of azithromycin is as effective as a standard seven-day, twice daily regimen of doxycycline in eradicating uncomplicated chlamydial genital infections. ${ }^{9-12}$ Thus, single dose treatment for $C$ trachomatis infections could be an important advance in the management and public health control of this significant disease.

We conducted this large randomised, openlabel comparative trial to determine whether a single $1 \mathrm{~g}$ oral dose of azithromycin was also safe and effective among women and men with uncomplicated chlamydial urogenital infection seen in a variety of outpatient clinical settings more closely resembling clinical practice.

\section{Methods}

Study population and procedures

Patients with presumptive chlamydial endocervicitis or urethritis were enrolled at 40 sites across the United States. Participants were at least 15 years of age and were recruited from a wide spectrum of outpatient settings, including sexually transmitted disease clinics, hospital-based gynaecology clinics, private practice offices, and college-student and adolescent health centres. A positive chlamydia antigen detection was required of all patients to be considered for bacteriologic efficacy. Patients with clinically apparent mucopurulent cervicitis or urethritis were eligible for enrolment only if they also had a positive urethral or cervical 
chlamydial antigen detection assay. Asymptomatic individuals who were sexual contacts of partners with proven chlamydial infection were also eligible for study entry. Subjects with a diagnosis of concomitant gonococcal infection required a positive chlamydial assay to be considered for enrolment. The rapid screening tests used at the various sites included Kodak Surecell ${ }^{\circledR}$ (Kodak, Rochester, NY), Ciba enzyme immunoassay [EIA] (Ciba-Geigy, Summitt, NJ) Chlamydiazyme ${ }^{\circledR}$ (Abbott Laboratories, Chicago, IL), Abbott Testpack $®$ (Abbott Laboratories, Chicago, IL), and Microtrak ${ }^{\circledR}$ direct monoclonal-fluorescent-antibody test (Syva Company, Palo Alto, CA).

Prospective patients were excluded from enrolment for the following reasons: clinical evidence of pelvic inflammatory disease; gonococcal pharyngitis, proctitis, or disseminated gonococcal infection; treatment with any systemic antibiotic with potential anti-chlamydial activity within 72 hours of enrolment; history of allergy or hypersensitivity to erythromycin, doxycycline, ceftriaxone, or any other betalactam antibiotic; pregnancy, lactation, or refusal to agree to use adequate contraception during and for three months following study completion; history of ulcers, gastrectomy, or other conditions possibly affecting drug absorption; or evidence or history of significant haematologic, renal, or cardiac disease.

After written informed consent was obtained, patients were interviewed regarding demographic variables and sexual and medical history. A directed physical examination, including pelvic examination, was performed, and all abnormal findings were recorded. Specimens for chlamydia testing were obtained using a urethral swab from males and a cervical or separate cervical and urethral swabs (or brushes) from females. Gram stains of smears obtained from the urethral and/or cervical secretions were performed to detect the presence of Neisseria gonorrhoeae. Laboratory evaluations included complete blood count (CBC) with differential and platelet counts; serum chemistry, including liver function tests; urinalysis; and a serologic test for syphilis. The treatment regimen administered was determined using a randomisation schedule which allocated azithromycin and doxycycline in a 2:1 fashion, respectively. The randomisation schedule and antibiotics were provided by Pfizer Labs, New York, NY. Patients assigned to receive azithromycin were administered the single $1 \mathrm{~g}$ dose by mouth as four $250 \mathrm{mg}$ capsules in the clinic, at least one hour before or two hours after a meal. Patients assigned to receive doxycycline were given $100 \mathrm{mg}$ capsules and were instructed to take one capsule every 12 hours for seven days. Patients with concomitant gonococcal infections were treated with a single $250 \mathrm{mg}$ intramuscular injection of ceftriaxone at the initial visit or at the first follow-up visit. While patients were asked to abstain from sexual activity during the study, instructions were given regarding condom use if they did have sexual intercourse.
Participants were asked to return for followup visits one week ( 5 to 9 days) and two weeks (12 to 18 days) after enrolment. Each followup visit included an assessment of drug compliance, interval history of symptoms, review of adverse experiences, intercurrent illness, and concomitant therapy; a genital examination with specific attention to signs relative to the initial visit; the collection of endocervical and/or urethral specimens for gram stain (if positive at enrolment) and non-culture chlamydia assay; and the collection of blood and urine for the standard laboratory tests performed at enrolment. The clinical response to treatment was classified in the following manner: complete resolution of signs and symptoms of infection (cure), incomplete resolution of signs and symptoms (improvement), and no apparent response or worsening of signs and symptoms (failure). Bacteriologic assessment of response was based on test results at the two-week follow-up visit, and was classified as either a negative chlamydial assay (cure) or positive assay (failure).

Patients with serological findings positive for syphilis at enrolment were discontinued from the study and treated, as were patients who were given additional antibiotics potentially active against $C$ trachomatis during the follow-up period.

Adverse events that occurred during therapy or within the two week period following enrolment were obtained by clinical and laboratory monitoring, by voluntary statements made by the study subjects, and by direct questioning. The severity of each adverse event was classified as mild, moderate, or severe.

\section{Statistical methodology}

Demographic variables were summarised and tested for differences between treatment groups. Categorical variables were tested using the Pearson's chi square test and continuous variables were tested using a one-way ANOVA. Clinical and assay response rates were tested for significant treatment group differences using the Pearson's chi square test. Analyses of clinical and assay response rates were stratified by gender using the CochranMantel-Haenszel test.

\section{Results}

Patient characteristics

Five hundred and ninety seven patients were enrolled at the 40 sites; 402 were randomised to receive azithromycin and 195 received doxycycline. Analysis of the baseline demographic data (table 1) showed no significant differences between the two treatment groups. Of the 597 patients who received study medication, $349(87 \%)$ azithromycin and 165 $(85 \%)$ doxycycline subjects completed the two-week study period. Eighty three enrolled participants discontinued or were dropped from study participation for a variety of reasons, including poor compliance with the study protocol (57), protocol violation (12), failure to meet entry criteria (8), and other reasons (6). 
Table 1 Selected characteristics of azithromycin and doxycycline groups at enrolment

\begin{tabular}{lcc}
\hline & $\begin{array}{c}\text { Azithromycin } \\
(N=402)\end{array}$ & $\begin{array}{c}\text { Doxycycline } \\
(N=195)\end{array}$ \\
\hline $\begin{array}{l}\text { Mean Age (years) } \\
\text { Range }\end{array}$ & $24 \cdot 4 \pm 7 \cdot 3$ & $23 \cdot 6 \pm 6 \cdot 7$ \\
$\begin{array}{l}\text { Sex, No (\%) } \\
\text { Female }\end{array}$ & $15-59$ & $13-53$ \\
$\quad$ Male & $257(64)$ & $118(61)$ \\
Race/ethnicity, No (\%) & $145(36)$ & $77(39)$ \\
$\quad$ Black & $206(51)$ & $109(56)$ \\
$\quad \begin{array}{l}\text { White } \\
\text { Other }\end{array}$ & $167(42)$ & $63(32)$ \\
Mean duration of & $29(7)$ & $23(12)$ \\
symptoms prior to & $9 \cdot 37 \pm 35 \cdot 5$ & $8 \cdot 72 \pm 16 \cdot 7$ \\
treatment (days) & $(\mathrm{N}=352)$ & $(\mathrm{N}=190)$ \\
\hline
\end{tabular}

Table 2 Chlamydia assay response to treatment with azithromycin and doxycycline at each follow-up visit *

\begin{tabular}{lcl}
\hline Treatment & $\begin{array}{l}\text { Positive } \\
\text { No (\%) }\end{array}$ & $\begin{array}{l}\text { Negative } \\
\text { No (\%) }\end{array}$ \\
\hline Week 1 & & \\
Azithromycin & $28(8)$ & $323(92)$ \\
Doxycycline & $18(11)$ & $146(89)$ \\
Week 2 & $9(3)$ & $338(97)$ \\
Azithromycin & $2(1)$ & $161(99)$ \\
Doxycycline & \\
\hline
\end{tabular}

^Includes only patients evaluable for bacteriological efficacy.

Table 3 Bacteriological response to treatment by nonculture assay cross-tabulated at Week $1 v$ Week 2

\begin{tabular}{llll}
\hline & & \multicolumn{2}{l}{ Week 2 response } \\
\cline { 2 - 4 } Treatment & Week 1 & Negative & Positive \\
& response & No (\%) & No (\%) \\
\hline Azithromycin & Negative & $298(91)$ & $2(0 \cdot 6) \dagger$ \\
& Positive & $21(6)^{\star}$ & $5(2) \ddagger$ \\
Doxycycline & Negative & $134(89)$ & $1(0 \cdot 7) \dagger$ \\
& Positive & $15(10)^{\star}$ & $0 \ddagger$ \\
\hline
\end{tabular}

^Resolving infection

tRelapse or reinfection.

$¥$ Treatment failure.

\section{Bacteriological efficacy of treatment}

A total of 351 azithromycin and 164 doxycycline subjects was evaluable for assay response at week one, and 347 azithromycin and 163 doxycycline subjects was evaluable for assay response at week two (two participants in each group who completed the two-week study period were not evaluable for bacteriological outcome owing to absence of assays). At the one-week follow-up visit, 28 azithromycin participants $(8 \%)$ and 18 doxycycline participants $(11 \%)$ had persistently positive chlamydia anti-

Table 4 Clinical response to treatment at each follow-up evaluation

\begin{tabular}{|c|c|c|c|c|}
\hline \multirow[b]{2}{*}{ Response } & \multicolumn{2}{|l|}{ Week 1} & \multicolumn{2}{|l|}{ Week 2} \\
\hline & $\begin{array}{l}\text { Azithromycin } \\
\text { No }(\%)\end{array}$ & $\begin{array}{l}\text { Doxycycline } \\
\text { No (\%) }\end{array}$ & $\begin{array}{l}\text { Azithromycin } \\
\text { No (\%) }\end{array}$ & $\begin{array}{l}\text { Doxycycline } \\
\text { No (\%) }\end{array}$ \\
\hline $\begin{array}{l}\text { Cured } \\
\text { Improved } \\
\text { Failed }\end{array}$ & $\begin{array}{c}162(61) \\
101(38) \\
3(1)\end{array}$ & $\begin{array}{c}70(60) \\
46(39) \\
1(1)\end{array}$ & $\begin{array}{c}225(86) \\
34(13) \\
2(1)\end{array}$ & $\begin{array}{c}96(83) \\
16(14) \\
4(3)\end{array}$ \\
\hline
\end{tabular}

Table 5 Clinical outcome of patients with positive chlamydia assay tests at Week 1 only versus patients with positive tests at Week 2 only, $n=34^{\star}$

\begin{tabular}{|c|c|c|c|c|c|c|}
\hline \multirow{2}{*}{$\begin{array}{l}\text { Clinical } \\
\text { Response }\end{array}$} & \multicolumn{3}{|c|}{ Positive/Week 1} & \multicolumn{3}{|c|}{ Positive/Week 2} \\
\hline & Azithromycin & Doxycycline & Total & Azithromycin & Doxycycline & Total \\
\hline $\begin{array}{l}\text { Improved/cured } \\
\text { Failed }\end{array}$ & $\begin{array}{r}19 \\
1\end{array}$ & $\begin{array}{r}14 \\
0\end{array}$ & $\begin{array}{r}33 \\
1\end{array}$ & $\begin{array}{l}6 \\
0\end{array}$ & $\begin{array}{l}1 \\
0\end{array}$ & $\begin{array}{l}7 \\
0\end{array}$ \\
\hline
\end{tabular}

*Includes only patients evaluable for clinical response at both Week 1 and Week 2 . gen detection tests. At the two-week follow-up evaluation, there were nine treatment failures (four males, five females) in the azithromycin group (3\%; 95\% confidence interval, 1 to $5 \%$ ) and two (one male, one female) in the doxycycline group ( $1 \%$; $95 \%$ confidence interval $<1$ to $4 \%$ ) (table 2 )

Treatment failures were manifested as persistently positive chlamydia assays at both follow-up visits. When the assay response was cross-tabulated at week one versus week two, five azithromycin and no doxycycline patients were considered failures by positive assay results at both the week one and week two evaluations (table 3). Additionally, two azithromycin patients and one doxycycline patient had relapsed or were reinfected between week one and week two (negative assay results at the first visit and a positive result at week two).

\section{Clinical response to therapy}

Subjects enrolled who were asymptomatic contacts (no signs of infection) of patients with proven chlamydial infection were not evaluable for clinical response to treatment. Among the symptomatic patients, 266 azithromycintreated patients (171 females and 95 males) and 117 doxycycline-treated patients (76 females and 41 males) could be assessed for clinical response to therapy at the first followup visit: 261 azithromycin-treated patients (170 females and 91 males) and 116 doxycycline-treated patients $(75$ females and 41 males) could be assessed at the second followup visit. For nearly two-thirds of the patients in both the azithromycin (61\%) and doxycycline $(60 \%)$ groups, symptoms were classified as resolved at the week one follow-up (table 4). At the second follow-up visit, the percentage of cured patients rose to $86 \%$ and $83 \%$ of the two respective groups, with an additional $13 \%$ and $14 \%$, respectively, showing improvement. Clinical outcomes were compared for patients who had positive assay tests at week one only and those who had positive tests at week two only (table 5). Only one patient of 34 with a positive test at week one had persistent signs of clinical infection compared to none of 7 at week two.

Concomitant infections with $N$ gonorrhoeae occurred in 31 patients who received azithromycin and 15 doxycycline-treated patients who were eligible for evaluation of bacteriological efficacy. All patients in both treatment groups were clinically cured or improved at the one week follow-up visit. At the week two visit, one patient who received doxycycline was reported as a clinical failure. She presented with clinical signs of cervicitis, including a mucopurulent endocervical exudate, cervical oedema, and erythema. Cervical oedema and endocervical exudate had been eliminated by week one but were present again at week two. She had received $250 \mathrm{mg}$ of ceftriaxone at enrolment for gonococcal cervicitis and denied interval sexual exposure during the follow-up period.

Treatment-related adverse events

One hundred sixty six (41\%) of the 402 study 
Table 6 Selected adverse events among treatment groups

\begin{tabular}{lcc}
\hline & \multicolumn{2}{l}{ No of patients (\%) } \\
\cline { 2 - 3 } Adverse event & $\begin{array}{c}\text { Azithromycin } \\
(N=402)\end{array}$ & $\begin{array}{l}\text { Doxycycline } \\
(N=195)\end{array}$ \\
\hline Gastrointestinal & $66(16)$ & $43(22)$ \\
Nausea & $36(9)$ & $8(4)$ \\
Diarrhoea & $9(2)$ & $8(4)$ \\
Vomiting & $11(3)$ & $5(3)$ \\
Dyspepsia & $23(6)$ & $8(4)$ \\
Abdominal pain & $14(3)$ & $1(<1)$ \\
Central Nervous System & $30(7)$ & $73(37)$ \\
Urogenital & $166(41)$ & \\
Total &
\end{tabular}

subjects who received azithromycin and 73 (37\%) of the 195 who received doxycycline reported at least one adverse event, most often involving the gastrointestinal tract (table 6). Nausea and diarrhoea were the most common azithromycin-related adverse events, although more patients taking doxycycline reported nausea $(22 \%)$ than azithromycin-treated patients $(16 \%)$. The central nervous system was the only organ system for which there was a significant difference in adverse events $(p=0.03)$ between treatment groups. Dizziness accounted for the highest number of reported events, seven $(2 \%)$ azithromycin patients versus one $(<$ $1 \%)$ doxycycline patient. All adverse events were judged to be of mild or moderate severity and resolved by the second follow-up visit.

Ten azithromycin-treated patients and two doxycycline-treated patients were reported to have elevations in results of liver function tests during the study. Two patients who received azithromycin had elevated liver enzymes at the second follow-up visit. One patient who received doxycycline and one azithromycin patient had elevated alanine aminotransferase (ALT) levels at their last study visits, but were lost to follow-up. Prior to the end of the study, all liver function abnormalities returned to normal in the remaining patients.

Two patients became pregnant while enrolled in the study; both received azithromycin. One patient elected to terminate the pregnancy for reasons other than study participation. The other patient carried the pregnancy to term and delivered a healthy infant.

\section{Discussion}

In this randomised, comparative trial, a single $1 \mathrm{~g}$ dose of azithromycin was as effective as the seven-day doxycycline regimen for the treatment of uncomplicated cervical and urethral infections caused by $C$ trachomatis. The outcomes observed for seven days of doxycycline therapy are consistent with the results reported in previous multicentre studies of uncomplicated chlamydial infections. ${ }^{1314}$ The observed cure rates in excess of $90 \%$ are likewise consistent with those from other reports of singledose therapy with azithromycin for treatment of uncomplicated chlamydial lower genital tract infections. ${ }^{9-12}$ Our study differs from other USA trials of azithromycin in two major ways: (1) the use of non-culture chlamydia detection tests to identify cases and to assess bacteriological outcome; and (2) the trial was conducted outside of the usual academic setting. Additionally, the final follow-up assessments were made only two weeks after enrolment, as opposed to the usually recommended four or more weeks.

In this study, nearly 600 patients were enrolled primarily at sites providing regular clinical care rather than at university-based research centres. The number and diversity of study sites were substantially greater in this trial than in an earlier reported study ${ }^{9}$ and serve to validate the efficacy of azithromycin in a "realworld" setting. The use of primary care settings outside of university centres, as in this study, provides evidence that azithromycin can be successfully used to treat chlamydia infection in such primary care settings. In comparison with the earlier study, a major difference in outcome in this study was that adverse events were approximately twice as common for both drugs. Whether this difference represents a true difference in the proportion of patients experiencing adverse events, a difference in how questions to elicit adverse events were asked, or simply differences in the populations studied is unknown. As there was no specified methodology for eliciting adverse events, some investigators may have been more intensive than others in their questioning of patients.

A second difference between this and earlier studies was the use of non-culture antigen detection assays for identification of cases and evaluation of bacteriological outcomes. While these tests are somewhat less sensitive than optimally performed cell cultures, logistical issues such as specimen transport, limited access to culture media, and cost considerations combine to make non-culture methods most likely to be used for chlamydia diagnosis in private offices and outpatient clinics. Among symptomatic women and men, their sensitivity ranges from $60-92 \% .{ }^{15}$ More recent generations of commercially available non-culture tests now have a specificity comparable to cell culture (98-100\%). Using a variety of commercially available rapid detection tests two weeks following initiation of therapy, bacteriological response to therapy in this study was $97 \%$ and $99 \%$ in azithromycin and doxycycline-treated patients, respectively. These results are very similar to previously published reports in which culture methods were utilised, primarily at university-based centres and suggest that the use of rapid non-culture diagnostic techniques for evaluating therapeutic regimens is a reasonable alternative when cultures for $C$ trachomatis are not available.

An additional concern which might be raised regarding this study would be the adequacy of the two-week follow-up for assessing response to therapy for chlamydial infections. Previous trials comparing ciprofloxacin to doxycycline for the treatment of nongonococcal urethritis revealed late treatment failures that were identified three weeks or more after completion of therapy by a sensitive culture system. ${ }^{16} 17$ Of some concern in this study was the consideration that false-negative tests of cure might arise if a treatment regimen simply reduced the number of organisms below the detection limit of an insensitive antigen detection assay. However, 
the results of the current study nearly parallel those of the previously published report, ${ }^{9}$ where virtually the same number of treatment failures was detected in the two and four week followup evaluations as at the one and two-week evaluations in the present study.

While further study is needed, our results suggest that follow-up using non-culture tests at two weeks after treatment accurately predicts bacteriological cure. Of interest were the 33 patients who were judged clinically cured but who had positive assay tests at week one. These cases most likely represent prolonged antigen excretion after apparent eradication of the organism, but they may be a source of confusion if a two week assessment of response to therapy using non-culture tests had not been used. However, given the high efficacy of both of these treatment regimens, the 1993 CDC STD Treatment Guidelines do not recommend routine use of test of cure. ${ }^{18}$

In contrast to gonococcal infections, where single dose treatment has long been available, until recently only multiple-dose regimens were recommended for treatment of chlamydial infections. These treatment regimens (tetracycline, doxycycline, and erythromycin given two to four times daily) have considerable potential for patient noncompliance. ${ }^{19}$ Noncompliance results from factors such as forgetfulness, misunderstanding of the importance of treatment, doubt about the diagnosis (especially in empiric treatment without laboratory testing), and asymptomatic infections. The last two factors are especially important with respect to chlamydial infections. Further, compliance in clinical practice is almost certainly less than that observed in clinical trials. One study revealed that the level of compliance deteriorates as the duration of treatment increases, and declines between clinic visits. ${ }^{20}$ Patient compliance is particularly relevant in the treatment of chlamydia, not only in preventing transmission of the disease, but also for prevention of subsequent complications. In addition, noncompliance may be more problematic when treating asymptomatic sex partners of symptomatic patients. Such asymptomatic contacts may be poorly motivated to seek medical attention, and even less inclined to take therapy. Although education and other behavioural initiatives should concentrate on compliance with multiple-dose regimens, the availability of single dose therapy for treatment of uncomplicated chlamydial infections marks a significant step toward disease control and reduced morbidity. Our data support an expanded role for azithromycin as single-dose therapy for uncomplicated chlamydial infection.

In addition to the authors, the Chlamydia One-Dose Azithromycin Trial Study Group consist of the following: D
Austin-Boulder, CO; J Bernstein-Dayton, OH; L Borgatta-
White Plains, NY; S Brunton-Long Beach, CA; J CareyOklahoma City, OK; S Doty-San Antonio, TX; C EricssonHouston, TX; J Estess-Hollandale, MS; R Farron-Far Rockaway, NY; R Galask-Iowa City, IA; V GennisMilwaukee, W; G Kent-San Jose, CA; E Kohorn-New Haven, CT; E Langston-Indianapolis, IN; H Lefler-Fort Worth, TX; M Loveless-Portland, OR; R Messer-Lubbock, TX; G Monif-Omaha, NE; G Noskin-Chicago, IL; R NussJacksonville, FL; M Pearlman-Ann Arbor, MI; P RayChicago, IL; J Reed-St Louis, MO; M Rein-Charlottesville, VA; R Santos-Chicago, IL; R Snow-Birmingham, AL; B
Snyder-New Brunswick, NJ; V Spagna-Columbus, OH; G Snyder-New Brunswick, NJ; V Spagna-Columbus, OH; G Tan-Akron, OH; A Thomas-New York, NY; J ToneyTampa, FL; J Turner-Columbia, SC; L Walker-Rochester, NY; D Whitson-Johnson City, TN; R Wodka-Hacienda Heights, CA

The research was supported by a grant from Pfizer Labs, New York, New York. The authors are indebted to Sheila Crout and Carol Read for editorial assistance in the preparation of this manuscript.

1 Washington AE, Johnson RE, Sanders LL Jr. Chlamydia trachomatis infections in the United States: what are they costing us? $¥ A M A$ 1987;257:2070-2.

2 Chow JM, Yonekura ML, Richwald GA, et al. The association between Chlamydia trachomatis and ectopic pregnancy: a matched-pair, case-control study. $¥ A M A$ 1990;263: a match

3 Patton DL. Experimental systems. In: Bowie WR, Caldwell $\mathrm{HR}$, Jones RB, et al, eds. Chlamydial infections: proceedings of the Seventh International Symposium of Human Chlamydial Infections, Harrison Hot Springs, BC, Cambridge, England: Cambridge University Press 1990:223-31.

4 Slaney $L$, Plummer R, Ronald AR, et al. In vitro activity of azithromycin (CP-62,993) against Neisseria gonorrhoeae, Haemophilus ducreyi, and Chlamydia trachomatis. $\mathcal{f}$ Chemother Infect Dis Malig 1989;1 (Suppl 1):400-3.

5 Slaney L, Plummer F, Ronald AR, et al. In vitro activity of azithromycin, erythromycin, ciprofloxacin, and norfloxacin against Neisseria gonorrhoeae, Haemophilus ducreyi, and Chlamydia trachomatis. $\mathcal{F}$ Antimicrob Chemother 1990; 25(Suppl A): $1-5$.

6 Foulds G, Shepard RM, Johnson RB. The pharmacokinetics of azithromycin in human serum and tissues. $\mathcal{F}$ Antimicrob Chemother 1990;25(Suppl A):73-82.

7 Ryan RW, Kwasnik I, Tilton RC. In vitro activity of macrolide CP 62993-XZ450 against selected microorganisms. In: Program and abstracts of the 26th Interscience Conference on Antimicrobial Agents and Chemotherapy, New Orleans, September 28-October 1, 1986. Washington, DC: American Society for Microbiology 1986:267.

8 Baldwin DR, Wise R, Andrew JM, et al. Azithromycin concentrations at the sites of pulmonary infection. Eur Respir $\mathcal{f}$ 1990;3:886-90.

9 Martin DH, Mroczkowski TF, Dalu ZA, et al. A controlled trial of a single dose of azithromycin for the treatment chlamydial urethritis and cervicitis. N Engl f Med 1992; 327:921-5.

10 Steingrimsson $\mathrm{O}$, Olafsson $\mathrm{JH}$, Thorarinsson $\mathrm{H}$, et al. Azithromycin in the treatment of sexually transmitted disease. F Antimicrob Chemother 1990;25(suppl A):109-14.

11 Van den Bosch C. Multicenter comparison of single dose azithromycin versus doxycycline in the treatment of sexually transmitted chlamydial infections. (Abstract 163). 6th International Congress for Infectious Diseases, Montreal Canada, July 1990.

12 Johnson RB. The role of azalide antibiotics in the treatment of chlamydia. Am $\mathcal{F}$ Obstet Gynecol 1991;164:1794-6.

13 Sanders LL Jr, Harrison HR, Washington AE. Treatment of sexually transmitted chlamydial infections. $\mathfrak{f} A M A$ 1986; 255:1750-6.

14 Toomey KE, Barnes RC. Treatment of Chlamydia trachomatis genital infection. F Infect Dis 1990;12(suppl 6) S645-55.

15 Stamm WE. Diagnosis of Chlamydia trachomatis genitourinary infections. Ann Intern Med 1988;108:710-7.

16 Hooten TM, Rogers E, Medina TG, et al. Ciprofloxacin compared with doxycycline for nongonococcal urethritis. JAMA 1990;264:1418-21.

17 Fong IW, Linton W, Simbul M, et al. Treatment of nongonococcal urethritis with ciprofloxacin. Am $\mathcal{F}$ Med 1987; 82(suppl 4A):311-6.

18 Centers for Disease Control and Prevention. 1993 Sexually transmitted diseases treatment guidelines. MMWR 1993;AZ(No RR-14):51.

19 Katz BP, Zwickl BW, Cain VA, et al. Compliance with antibiotic therapy for Chlamydia trachomatis and Neisseria gonorrhoeae. Sex Transm Dis 1992;19:351-4.

20 Cramer JA, Scheyer RO, Mattson RH. Compliance declines between clinic visits. Arch Intern Med 1990;150:1509-10. 